\title{
Atención y craving o ganas compulsivas. Avances en su conceptualización y su implicación en la prevención de recaídas
}

\author{
Jesús Blasco*; José Martínez-Raga*， **; Emelia Carrasco***; Javier Didia-Attas**** \\ * Unidad de Conductas Adictivas Departamento de Salud de Gandía, Agencia Valenciana de Salud (España). \\ * * Departamento de Fisiología, Farmacología y Toxicología, Universidad CEU Cardenal Herrera, Valencia (España). \\ *** Práctica privada, Valencia (España). \\ ****Hospital Italiano de Buenos Aires (Argentina) \\ Enviar correspondencia a: \\ Jesús Blasco Gallego. Centro de Salud de Corea. Unidad de Conductas Adictivas. C./ Benissuai 22, 46700 Gandia, Valencia (España) \\ Tel: +34 96 2959736. Facsimile: +34 96 2959709. E-mail: jblasco070d@cv.gva.es
}

Recibido: Septiembre de 2007 Aceptado: Julio de 2008

\section{RESUMEN}

El craving, ganas compulsivas, deseo intenso o compulsión irrefrenable a consumir es uno de los síntomas centrales en el síndrome de dependencia y de gran importancia en las recaídas al abuso de alcohol u otras drogas. El presente trabajo revisa la relación entre la atención, los procesos atencionales y el fenómeno del craving. Se abordan los diferentes elementos integrantes de la atención y en particular de la relevancia de la atención selectiva sobre el craving y las etapas iniciales de la recaída. Se revisan las estrategias psicológicas eficaces frente al craving, en especial en el modelo cognitivo conductual de la recaída y en la terapia de prevención de recaídas. Finalmente, se discuten las características de la denominada terapia atencional, basada en el "mindfulness" o "atención consciente" y su potencial utilidad en el abordaje de los trastornos adictivos en general y en el craving en concreto.

Palabras clave: craving, atención, atención selectiva, prevención de recaídas, mindfulness.

\section{ABSTRACT}

Irresistible compulsion to use, or craving, is one of the key symptoms of substance dependence syndrome, and of major relevance with regard to relapse in the abuse of alcohol or other drugs. In the present paper, the relationship between attention, attentional processes and the craving phenomenon is reviewed. The paper also explores the different elements of attention, and particularly the relevance of selective attention in craving and the initial stages of relapse. Those psychological strategies that have emerged as effective in the management of craving for substances are also reviewed, with particular emphasis on the cognitive-behavioral model of relapse and on relapse-prevention therapy. Finally, we discuss the characteristics of so-called attentional therapy, based on mindfulness, and its potential effectiveness in the management of addictive disorders in general and of craving in particular.

Key words: craving, attention, selective attention, relapse prevention, mindfulness.

\section{INTRODUCCIÓN}

E fenómeno del craving, ansia, ganas compulsivas, deseo intenso o compulsión irrefrenable de consumir una sustancia psicótropa o de experimentar sus efectos intoxicantes ${ }^{1}$ es uno de los elementos centrales en la mayoría de los modelos teóricos de los trastornos adictivos. Es además uno de los criterios diagnósticos esenciales del síndrome de dependencia dentro de la décima edición de la Clasificación Internacional de las Enfermedades (ClE-10)². En la CIE-10 se define el craving o ganas compulsivas como "deseo intenso o vivencia de una compulsión a consumir una sustancia psicótropa o de experimentar sus efectos tóxicos. El deseo es intenso y puede originarse ante la presencia de la sustancia, su olor o sabor, o ante otros estímulos que hayan estado asociados a su consumo en el pasado".

El fenómeno craving se ha intentado definir y explicar desde diferentes enfoques. Los modelos neurobiológicos ${ }^{3,4,5}$ han intentando identificar el sustrato neurofisiológico del craving y porqué este persiste durante largos períodos de tiempo. Desde las teorías del aprendizaje, los modelos basados en el condicio- 
namiento operante ${ }^{6}$ tratan de explicar el deseo en función de los refuerzos contingentes a la conducta de consumo que incrementan la probabilidad de esta, mientras que los modelos basados en el condicionamiento clásico ${ }^{7}$ explican el mantenimiento del deseo a largo plazo en base a la exposición repetida a la droga. Desde la aproximación cognitivista, dos modelos fundamentales tratan de abordar el craving: modelos de aprendizaje social ${ }^{8,9}$ que enfatizan constructos como expectativas, atribuciones, imitación y autoeficacia y modelos de la ciencia cognitiva ${ }^{10,11,12}$ centrados en el procesamiento de la información, estilos cognitivos, memoria y toma de decisiones.

Hasta la aparición de los modelos cognitivos, el craving ha constituído un elemento esencial a la hora de explicar la pérdida de control y la ejecución de la conducta de consumo, asumiendo la experimentación de dicho fenómeno en los pacientes drogodependientes antes de un primer consumo o de una recaída13, 14, aunque con diferencias respecto a las distintas sustancias tóxicas ${ }^{15}$.

Modelos más recientes, especialmente el basado en la teoría del Procesamiento Cognitivo de Tiffany ${ }^{12}$ apuntan a un desplazamiento del fenómeno "craving" como elemento central explicativo de la conducta de consumo. Según este modelo, el sujeto adicto podría desarrollar la conducta de consumo bien bajo procesos cognitivos de carácter automático, bien bajo procesos cognitivos de carácter controlado. Sería bajo la influencia de los procesos cognitivos de carácter controlado cuando aparecería en el adicto la experiencia subjetiva de craving, pudiendo no aparecer bajo la influencia de los procesos automáticos. El craving se experimentaría ante la puesta en acción de procesos no automáticos o controlados que el sujeto pondría en marcha ante dos situaciones posibles: encontrar dificultades para consumir por no disponer de la sustancia o intentar oponerse a la ejecución automática del consumo ${ }^{16,17}$. Estaríamos, según este modelo, ante conductas de consumo que podrían ser ejecutadas sin la experiencia de craving por parte del sujeto, lo que dificultaría enormemente la detección de las situaciones de riesgo de recaída. Así pues el craving sería un epifenómeno de las conductas adictivas, por lo que, ni sería absolutamente irrelevante, ni plenamente responsable para la comprensión de las toxicomanías ${ }^{18}$. No existe pues, un consenso total a la hora de correlacionar craving y consumo de drogas, pero parece claro que dicho fenómeno pueda estar implicado de forma bastante generalizada en los procesos de recaída, siendo necesario abordarlo en el tratamiento de deshabituación en conductas adictivas.

El presente trabajo intenta revisar los conocimientos teóricos y prácticos basados en las evidencias científicas en relación con craving o ganas compulsivas, todo ello desde el punto de vista de los procesos atencionales, con el objetivo de seguir avanzando en la construcción de modelos conceptuales sobre el fenómeno del craving, tratando así de detectar los factores concretos que pueden facilitar o entorpecer la consecución de la abstinencia. Así mismo, se pretende repasar los abordajes psicoterapéuticos que se han mostrado eficaces en el abordaje del craving desde el enfoque de los procesos atencionales, con especial atención a los procedimientos de "mindfulness". No es el objetivo de este artículo revisar todos los modelos explicativos del craving, sino abordar los aspectos que puedan ampliar su conceptualización y aportar alguna consideración que pudieran ser útiles para poder mejorar los tratamientos de deshabituación en la práctica clínica.

\section{LA ATENCIÓN}

\section{Problemas en su definición y concepto}

El estudio de la atención, desde los inicios de la psicología como ciencia, ha pasado por diferentes etapas, intentando ser explicada desde distintos modelos teóricos. Las primeras concepciones, predominantemente mentalistas ${ }^{19}$, fueron posteriormente desterradas por el paradigma conductista y recuperadas a su vez por la corriente cognitiva a través de la teoría del procesamiento de la información. Los clásicos modelos de filtro ${ }^{20}$ dieron paso a otros tipos de propuestas: los modelos de recursos atencionales ${ }^{21}$, el conexionis$\mathrm{mo}^{22}$, los modelos de automaticidad y de control atencional que se reseñan más adelante y que tratamos de relacionar con el funcionamiento del craving, hasta las últimas aportaciones de las neurociencias ${ }^{23}$.

La literatura especializada ofrece múltiples definiciones del concepto de atención, sin que hasta la fecha exista un consenso sobre los aspectos definitorios específicos de dicha dimensión 24, 25, 26, 27, 28. 29. Algunos autores hablan incluso de la posibilidad de "variedades de la atención"30. Es a partir de los años 70 cuando la atención comienza a adquirir relevancia como elemento explicativo de los procesos mentales. Los modelos que surgen comienzan a aportar datos sobre la importancia de la atención en el control de la ejecución de los procesos mentales ${ }^{31,32,33}$.

\section{La atención: ¿agente o resultado?}

La atención es una facultad que se ha asociado generalmente con los procesos de selección de la información y de control sobre los pensamientos y las conductas ${ }^{34}$, aunque la explicación a dichos procesos ha dependido de consideraciones teóricas sobre la atención. ¿Es la atención un agente causal o un resultado? La atención como agente causal sería 
el mecanismo responsable de desencadenar la puesta en marcha de todos los recursos necesarios para procesar la información, así de cómo estos recursos se van sucediendo y articulando en cada momento. Según esta aproximación teórica la atención tendría una posición central en el procesamiento cognitivo. La atención como resultado se entendería como un producto de otras instancias cognitivas de las cuales ella dependería, y a través de las que podría ser explicado su funcionamiento ${ }^{35}$. Este tema, actualmente muy debatido, no ha encontrado todavía un consenso.

Las conceptualizaciones teóricas actuales apuntan más hacia la atención como agente del que dependen los mecanismos de procesamiento de la información ${ }^{23}$, 29,36, dándole una posición eminentemente explicativa del funcionamiento mental. De este modo, la atención estaría mediando en el control de la conducta, a través de la regulación de los procesos mentales. Sería pues, desde estas concepciones nucleares, el mediador funcional indispensable de todos los procesos $\operatorname{cognitivos}^{37}$. En este sentido la atención se ha considerado como un mecanismo central de capacidad limitada que orienta y controla la actividad consciente del organismo de acuerdo con un objetivo determinado $^{28}$. En definitiva, la atención determinaría, de forma inevitable, la eficiencia del rendimiento en cada tarea cognitiva $^{38}$.

\section{La atención:¿consciente o inconsciente?}

La atención, ¿se genera de forma consciente o puede ocurrir inconscientemente?. Como en casi todas las áreas de estudio en el campo de la atención no existe consenso al respecto. Para algunos autores atención y consciencia serían una misma cosa ${ }^{28,}$ 32, 39 mientras que para otros la información atendida, podría no ser procesada conscientemente ${ }^{40}$. En cualquier caso, atención y consciencia no parecen ser sinónimos ya que no todo lo atendido se hace consciente. Todos tenemos ejemplos cotidianos, en los que ejecutamos conductas habituales sin prestar una atención consciente, como cambiar de marcha en nuestro coche. Este tipo de atención, que se suele llamar atención habitual, sería característicamente automática e inconsciente. Sin embargo, podemos afirmar que todo lo que es consciente ha sido atendido, es decir, las cosas ingresan en nuestra conciencia porque las atendemos, porque la atención se formaliza conscientemente. Todo ello ha conducido al desarrollo de modelos neuropsicológicos de la atención que intentan explicar las funciones de la misma a través de redes cerebrales que conectan diferentes áreas en el cerebro. Estos modelos implican el funcionamiento de dichas redes con la atención selectiva y cómo se estructura de una forma consciente lo que experimentamos en la vida 23,28
En base a lo mencionado, y de cara a la posible relación entre los procesos atencionales y las ganas compulsivas o craving por las drogas, se plantea la cuestión de si el procesamiento de la información se realiza en base a procesos automáticos o a procesos controlados. La literatura especializada actual indica que no se puede hablar de procesos totalmente automáticos o totalmente controlados ${ }^{41,42}$, aunque hace referencia a que la mayoría de los procesos que tienen un carácter automático, en un principio fueron ejecutados de forma controlada, siendo por tanto procesos mixtos ${ }^{43,44}$, localizados dentro de un continuum y que se diferenciarían por características de grado más que en sus características cualitativas ${ }^{45,46}$.

Pero, ¿cómo llega un proceso a automatizarse?, ¿qué factores están implicados en la adquisición de un automatismo?, ¿cómo llegan a automatizarse tareas que en un principio se ejecutan de forma controlada? Esta cuestión ocupa gran importancia en los principales estudios que tratan de abordar los procesos automáticos y controlados, señalando que es la práctica el factor que más sustento proporciona al proceso de automatización ${ }^{47,48,49}$.

\section{Atención selectiva}

Toda la información que nos llega, tanto del medio externo, como la generada en nuestro medio interno, necesita ser seleccionada con el objetivo de acomodar nuestras acciones a la consecución de los objetivos propuestos. A través de esta selección, tomamos determinadas decisiones para dar respuesta a las demandas del ambiente que son para nosotros importantes o relevantes. La selección atencional, tiene por tanto, la función de adaptarnos en nuestra interrelación con el medio.

La selección atencional ejecuta en paralelo dos procesos distintos cualitativamente, pero esencialmente iguales desde un punto de vista cuantitativo:

"Atender selectivamente a un estímulo o a algún aspecto del mismo, con preferencia sobre los otros estímulos"21, es decir, centrarse de forma muy concreta en determinados aspectos de nuestro entorno, así como específicamente en estímulos interioceptivos, con el objetivo de ejecutar una respuesta concreta. Es lo que comúnmente se denomina focalización, o más concretamente atención focalizada.

Al mismo tiempo que se ejecuta dicha focalización, se realiza un proceso de ignorar el resto de información considerada en esos momentos como no relevante, ya que el medio envía gran cantidad de información que requiere ser procesada para la elección y ejecución de una respuesta. En este contexto cobran especial relevancia las denominadas respuestas incompatibles, es decir, aquellas que debemos 
inhibir con el objetivo de poder ejecutar las respuestas relevantes, así como los estímulos distractores o no relevantes, los cuales interfieren notablemente sobre la capacidad de procesar la información por provocar oscilaciones de la focalización atencional ${ }^{50,51}$.

Cuando un sujeto fija voluntariamente la atención sobre un estímulo, ya sea interno o externo, estaría concentrando la atención, es decir se estaría produciendo una focalización atencional selectiva consciente. Por el contrario, cuando se fija la atención de forma involuntaria, hablaríamos de un proceso de focalización atencional selectiva no consciente, no controlada, con las implicaciones que ello puede tener en cuanto a la toma de decisiones y conductas a ejecutar por el sujeto.

\section{EL PROCESO DE LA RECAÍDA}

\section{Modelo Cognitivo-Conductual de la recaída}

Probablemente, es el modelo cognitivo-conductual de Marlatt ${ }^{13}$, el que goza hasta el momento de una mayor aceptación general para explicar el fenómeno de la recaída. Dicho modelo subraya la existencia de factores tanto personales como situacionales y presenta la recaída como una pérdida de control ante una situación de alto riesgo. Las situaciones de riesgo se aglutinan en tres grupos principales ${ }^{52,53,54}$ : Estados emocionales negativos, conflictos interpersonales y presión social (véase la figura 1). Si el sujeto es capaz de dar una respuesta de afrontamiento eficaz ante la situación de alto riesgo podrá elevar la probabilidad de evitar la recaída y aumentar su percepción de control.

Pero, ¿qué sucede cuando el adicto no ha sido capaz de afrontar las situaciones de alto riesgo? Esto se ha atribuido a la posibilidad de que el sujeto no haya adquirido nunca las capacidades de afrontamiento necesarias, o que las respuestas apropiadas estén inhibidas por el miedo o la ansiedad, de modo que el individuo no reconozca la situación como peligrosa; así mismo los déficits de motivación también minarían las estrategias de afrontamiento adquiridas ${ }^{55}$. Pero, uno de los aspectos más significativos es que "el aliciente de la gratificación inmediata se convierte en la figura dominante del campo perceptivo y la realidad de las consecuencias completas del acto pasa a un segundo término"13. Con frecuencia, como refiere Marlatt, "el individuo anticipa los efectos positivos inmediatos de la actividad (consumir la sustancia), una anticipación fundamentada en experiencias anteriores, mientras ignora o no presta atención a las consecuencias negativas involucradas a más largo plazo. Estas expectativas de las consecuencias positivas son un determinante principal del abuso de sustancias"56.

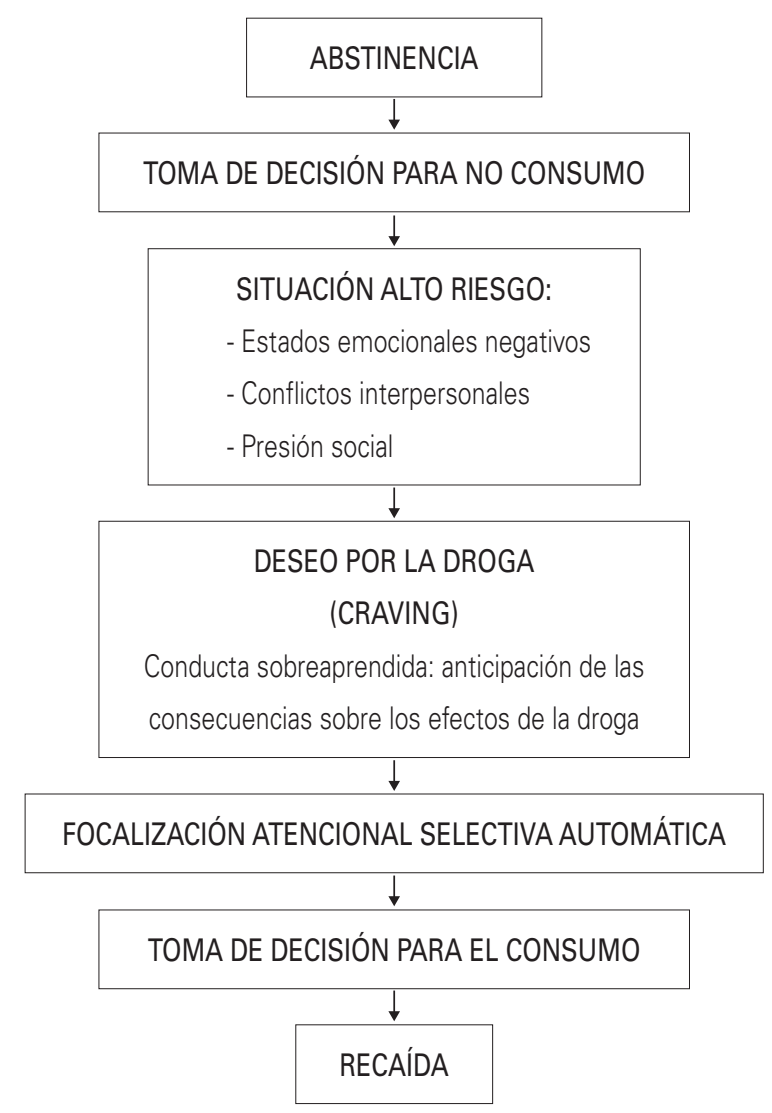

Figura 1. Esquema cognitivo-conductual de la recaída, al que se añade la "focalización atencional selectiva automática" como elemento explicativo y necesario para que se produzca la misma.

Esta forma de atención, prestar atención a los efectos reforzantes y a la vez no prestar atención a las consecuencias negativas, se convierte en uno de los factores explicativos más importantes, aunque no el único, del proceso de recaída en los adictos.

Ante esta forma particular de atención durante el episodio de craving, y siempre en base a la observación y a la experiencia clínica con pacientes adictos, surgen las siguientes cuestiones: ¿qué sucede en la mente del paciente para que su campo perceptivo se reduzca exclusivamente a los efectos reforzantes de la droga?; ¿qué mecanismo cognitivo se pone en funcionamiento provocando tal estrechamiento del campo perceptivo?

Cuando dirigimos nuestra atención hacia nuestros objetivos e inhibimos respuestas inapropiadas se ponen en marcha los mecanismos de la atención voluntaria. En estos casos decimos que tenemos un gran control atencional y exige un cierto esfuerzo para mantenerlo. Pero también existe una atención involuntaria que tiene lugar sin que medie proceso volitivo alguno. La experiencia relatada por los pacientes, tras una caída en el consumo o después de una recaída, confirma que durante el episodio de ganas compulsivas se produce una atención selectiva de carácter 
automático (no consciente) sobre los efectos "beneficiosos" que se derivan del consumo y una enajenación sobre los efectos "perniciosos", conduciendo así a una toma de decisión para el consumo:

"...es como si mi cabeza se hubiera cambiado, como si en esos momentos no fuera yo ..."

"...no se lo que me ocurrió. Cuando me di cuenta ya había consumido ..."

“..., aunque no tenía la intención de hacerlo, al final pudo conmigo. ..."

Parece poco razonable pensar que la conducta de consumo se formaliza sin una toma de decisiones en ese sentido, pero, ¿es posible una decisión distinta cuando sólo se dispone en la conciencia de los efectos reforzantes de la sustancia? O, lo que es lo mismo ¿es posible evitar una caída en el consumo o la recaída si la atención está "atrapada" por los efectos beneficiosos? Durante este proceso de focalización atencional selectiva automática que ocurriría en la mente del adicto, en el episodio de craving, la capacidad atencional consciente quedaría bloqueada, "atrapada", por los pensamientos sobre los efectos reforzantes del consumo ("consume y estarás bien"), así como por todos aquellos pensamientos encaminados a justificar la conducta de consumir ("es la última vez"; "nadie lo va a saber"; "yo ya lo controlo", etc.). Estos datos apoyarían la hipótesis de que la focalización atencional selectiva automática (déficit de atención consciente), durante el episodio de craving, actuaría como un potente factor de riesgo en la recaída, siendo pertinente la exploración y evaluación diagnóstica de este aspecto en los pacientes adictos.

Si el craving o ganas compulsivas pudiera ser considerado como epifenómeno de la conducta adicti$\mathrm{va}^{16,18}$, la focalización atencional selectiva automática sobre los efectos reforzantes de la droga sería el epifenómeno de la pérdida de control.

Nuestra hipótesis es que, únicamente si durante el episodio de craving el individuo es capaz de mantener en su conciencia la información disponible en su memoria a largo plazo respecto a todos los efectos (positivos y negativos) de su posible consumo, será posible que operativice una toma de decisiones "libre" respecto a su consumo o no. En este sentido, se ha distinguido entre procesos cognitivos automáticos y no automáticos, para señalar que la conducta adictiva, como un producto del aprendizaje a largo plazo, se podrá iniciar y completar sin la presencia de intencionalidad y por lo tanto con escaso esfuerzo cognitivo $^{12,16,57}$. Dada la rapidez y autonomía de la puesta en marcha de los procesos cognitivos que operan en la misma, será difícil inhibirlos, siendo necesario un esfuerzo cognitivo relevante.

Considerando lo expuesto, sería lógico plantear la necesidad de implementar estrategias encaminadas a incrementar la capacidad del paciente sobre el control atencional dentro de los propios programas de PR.

\section{Abordaje terapéutico de la recaída}

Diversas intervenciones farmacológicas se han evaluado para la prevención de recaídas en los trastornos adictivos, pudiéndose encuadrar en cuatro grandes grupos: tratamientos de sustitución, terapia con antagonistas, agentes aversivos y fármacos que disminuyen el deseo o craving. Se ha evidenciado la eficacia y utilidad de los tratamientos de sustitución con agonistas opiáceos para la disminución y la abstinencia mantenida en la dependencia de heroína ${ }^{58,59,60 ;}$ sin embargo, el uso de fármacos antagonistas como la naltrexona, parece reservado para pacientes adictos a opiáceos muy motivados y en combinación con terapias psicosociales ${ }^{61}$. De momento no se disponen de datos concluyentes respecto a estrategias farmacológicas eficaces para disminución de la probabilidad de la recaída en pacientes dependientes de cocaína ${ }^{62,63,}$ 64 . Las terapias farmacológicas aversivas han sido utilizadas frecuentemente en la dependencia de alcohol, especialmente el disulfiram, señalándose la posible utilidad en determinada tipología de paciente alcohólico ${ }^{65}$, como así lo son también determinados fármacos anticraving ${ }^{63,66}$. Con respecto a la dependencia de la nicotina, recientes revisiones sistemáticas con metaanálisis han evidenciado que el tratamiento con varenicline, un agonista parcial del receptor nicotínico, triplica la probabilidad de dejar de fumar ${ }^{67}$. Mediante fármacos como el bupropion o las terapia sustitutivas con nicotina, se aumenta las probabilidad de mantener la abstinencia tabáquica entre 1,5 y 2 veces ${ }^{66,68}$.

Las intervenciones psicológicas que han sido evaluadas con mayor frecuencia para demostrar su eficacia en la prevención de recaídas en el consumo de drogas han sido las que se encuentran bajo el enfoque cognitivo conductual. Las estrategias de evitación durante las fases iniciales de la abstinencia y las estrategias cognitivas a más largo plazo parecen ser eficaces frente a la recaída ${ }^{69}$ Los programas cognitivos-conductuales, especialmente los basados en entrenamiento de habilidades, son considerados hoy en día como de primera elección en el tratamiento de la dependencia alcohólica ${ }^{53,66,70,71,72 ; ~ e l ~ m a n e j o ~ d e ~}$ contingencias asociado al tratamiento farmacológico, se ha mostrado como un abordaje eficaz para la prevención de recaídas en pacientes adictos a la heroína u otras sustancias ${ }^{73,74}$. En cuanto a la dependencia de la cocaína, las terapias cognitivo-conductuales, están consideradas por el momento como tratamientos probablemente eficaces $66,71,75,76$, considerándose al Programa de Reforzamiento Comunitario más Terapia de Incentivos un abordaje bien establecido para prevenir la recaída a dicha sustancia ${ }^{77,78}$. Los tratamientos mul- 
ticomponentes que incluyen terapias conductuales modales parecen ser de elección en el tratamiento de la dependencia de nicotina ${ }^{66,68,79}$.

Dentro de los programas cognitivo-conductuales, se evidencia como abordaje eficaz el modelo de Prevención de Recaídas (PR) bien como tratamiento principal, o como tratamiento co-adyuvante ${ }^{53,66,72}$. Se ha descrito una reducción significativa de los consumos y una mejor evolución a largo plazo con el tratamiento de PR tanto para pacientes dependientes de alcohol80, 81, individuos con abuso o dependencia de cocaína ${ }^{82,83}$, para aquellos con adicción a cannabis, o para pacientes dependientes de opiáceos ${ }^{84,85}$.

La PR es un abordaje terapéutico en el que a partir de técnicas cognitivo-conductuales se intenta ayudar a los pacientes a desarrollar un mayor autocontrol que les permita anticipar y afrontar los problemas de las recaídas en el proceso de cambio de las conductas adictivas ${ }^{55}$. Entre las estrategias específicas de la PR se incluyen: discusión de la ambivalencia sobre el trastorno adictivo, identificación de desencadenantes emocionales y ambientales del craving y del consumo, desarrollo y revisión de estrategias específicas para hacer frente a los factores de estrés internos o externos, explorando la cadena de decisiones que conducen a la reanudación del consumo, aprendizaje de los episodios breves de consumo para explorar los desencadenantes de la posible recaída, desarrollo de técnicas eficaces para la intervención precoz, reducción de la exposición a estímulos relacionados con las drogas y modificación de las respuestas a los mismos ${ }^{52,53}$ 66. El modelo de PR tiene, por lo tanto, como objetivo primordial aumentar la probabilidad de que el sujeto adicto sea capaz de incrementar su autoeficacia ante las situaciones o estímulos dicriminativos hacia el consumo, ya tengan un carácter exterioceptivo o interioceptivo. En este modelo, el consumo, o incapacidad para hacer frente al deseo, ganas compulsivas o craving por la droga, es considerada como una conducta sobreaprendida, basada en la teoría que los procesos de aprendizaje desempeñan un papel esencial en el desarrollo de patrones de comportamiento de inadaptabilidad.

\section{MINDFULNESS YTERAPIA ATENCIONAL}

A lo largo de las últimas tres décadas ha surgido un creciente interés por las psicoterapias basadas en el "mindfulness", en parte a partir de los estudios de investigación que sugieren la utilidad de la meditación en las adicciones y en otras áreas de la salud mental ${ }^{86,}$ 87. Sin embargo, a pesar de que el concepto de "mindfulness" tiene su origen en tradiciones filosóficas, culturales y espirituales de tipo contemplativo, con particular influencia del budismo tibetano, la aplicación de las técnicas de "mindfulness" puede realizarse de forma efectiva sin tener en cuenta un vocabulario o una tradición cultural, espiritual, religiosa, o filosófica en particular ${ }^{88}$. Han sido varios los intentos de definir operacionalmente el concepto "mindfulness"88, 89, 90, ${ }^{91}$ y aunque no existe consenso sobre la traducción más apropiada al castellano, se han propuesto términos como "presencia atenta y reflexiva" o "atención y consciencia plena" para dicho término anglosajón. Nosotros pensamos que "mindfulness" se traduciría más adecuadamente como "atención consciente". Pero, atención consciente, ¿sobre qué? El "mindfulness" se define por prestar atención intencionadamente, en el momento presente, y sin prejuicios; es decir, tomando conciencia y prestar atención de un momento actual a otro ${ }^{88,92,93}$. Los términos temporales, pasado, presente y futuro, únicamente pueden ser manejados en el momento presente, por lo que sólo pueden ser operativizados en el presente. La adopción de una postura interior en la que los acontecimientos, siempre presentes, son observados sin valoración ni juicio, permitiría la objetivación de los mismos (un sentimiento o un pensamiento serían vivenciados como un objeto emocional o un objeto mental). Desde esta situación privilegiada, la percepción de la realidad interna del sujeto tiene un carácter menos distorsionado, menos sesgado, menos interpretado. La persona es capaz de ir vivenciando su mundo interno, de forma progresiva, como a "una cierta distancia". El lugar donde el paciente está, el lugar donde se vivencia a si mismo, no es el lugar donde se generan sus pensamientos y emociones. Las terapias basadas en el "mindfulness" o "atención consciente", tiene entre sus objetivos, la detección de dichos contenidos desde una actitud de aceptación (no valoración) de los mismos, tal y como son.

Otra característica y a la vez, uno de los objetivos del "mindfulness" es la ausencia por parte del sujeto del control de sus reacciones, sentimientos o pensamientos. No se trataría de reducir (controlar) el malestar, el miedo, la ira, la tristeza o el craving, según el caso. Más bien consistiría en que, manteniendo dicha sintomatología en observación, fueran los mecanismos naturales de autorregulación del propio individuo, los que ejercieran el control indirecto de reducción de los síntomas. Ante ello, y desde la óptica de la psicología clínica aplicada a los trastornos adictivos, nos formulamos la siguiente pregunta: ¿Qué podría suceder en pleno episodio de craving, si aún a pesar de una buena detección de los síntomas del mismo (físicos, emocionales y mentales), el adicto mantiene "simplemente" en observación dichos síntomas? ¿Cuánto tiempo será capaz de mantener en "observación sin manipulación" dichos síntomas y sin que ello conduzca a la pérdida de control (consumo)?

Desde esta perspectiva, cada vez más autores contemplan el "mindfulness" o "atención conscien- 
te" como un complemento de otros abordajes terapéuticos para la prevención de la recaída en el abuso de sustancias, compatibles y asociadas o insertas en psicoterapias de más amplio espectro ${ }^{94}, 95$, en especial en aquellas de orientación cognitivo-conductual. Los procedimientos o técnicas "mindfulness", están siendo incorporados a distintas psicoterapias denominadas de tercera generación, bien como programas terapéuticos completos como la denominada Mindfulness-based stress reduction (MBSR)96, 97, bien como componentes de las mismas como en el caso de la Terapia Dialéctico Conductual para Trastorno Límite de la Personalidad (DBT) ${ }^{98}$, la Terapia Cognitiva de la Depresión basada en Mindfulness ${ }^{99}$ o la Terapia de Aceptación y Compromiso (ACT) ${ }^{100}$

La medición de la "atención consciente" o "mindfulness" ha sido desarrollada recientemente a través de varios instrumentos, entre los que se incluyen: el Freiburg Mindfulness Inventory (FMI)101, la Mindful Attention Awareness Scale (MAAS) ${ }^{102}$, el Kentucky Inventory of Mindfulness Skills (KIMS) ${ }^{103}$, el Revised Cognitive and Affective Mindfulness Scale (CAMS$R)^{104}$, el Philadelphia Mindfulness Scale (PHLMS) ${ }^{105}$, y más recientemente la Toronto Mindfulness Scale ${ }^{106}$. Hasta el momento, se desconoce la adaptación y validación de pruebas diagnósticas para la medición de "mindfulness" o "atención consciente" en nuestro país.

Las terapias basadas en el "mindfulness" o "atención consciente" han sido evaluadas ya en varios estudios con pacientes con diversos trastornos adictivos $^{94,107,108,109,110}$. Sin embargo, las evidencias iniciales de la posible utilidad de este tipo de técnicas en el abordaje de los trastornos adictivos proviene de un estudio ya clásico en el que se observó que el $40 \%$ de un grupo de 126 sujetos que practicaban meditación trascendental al menos 2 años habían dejado de beber en los primeros seis meses, frente a ninguno de los 90 sujetos del grupo control'111. Asi mismo, un estudio piloto aleatorizado, la evolución de 18 pacientes con abuso de sustancias que durante 8 semanas recibieron terapia de "mindfulness" más tratamiento habitual fue comparada con la de otros 13 pacientes que recibieron tratamiento habitual, observándose una evolución global levemente más favorable en el grupo que recibió entrenamiento de "mindfulness"107. Otro estudio más amplio evaluó la efectividad de una forma de terapia basada en el "mindfulness", la meditación Vipassana (MV) en comparación con el tratamiento habitual, sobre el consumo de sustancias y una serie de variables psicosociales en una muestra de 173 reclusos con diversos trastornos adictivos. A los tres meses tras salir de prisión, aquellos sujetos que participaron en la terapia de MV presentaron una reducción significativa en el consumo de alcohol, de marihuana y de cocaína crack, así como una disminución en los problemas relacionados con el alcohol y en los síntomas psiquiátricos junto con un mejor funcionamiento psicosocial en comparación con los reclusos que recibieron el tratamiento habitual incluidos en el grupo control ${ }^{94,108,110}$. Más recientemente se ha estudiado una terapia de reducción de stress basada en el "mindfulness", aplicada en sesiones semanales de terapia grupal, en el tratamiento para dejar de fumar en un estudio piloto con 18 sujetos dependientes de nicotina ${ }^{109}$. A las seis semanas de la fecha prevista para dejar de fumar 10 de los 18 sujetos (56\%) mantenían abstinencia continuada de la nicotina confirmada mediante carboximetría; de hecho el cumplimiento con la meditación se asociaba positivamente con la abstinencia tabáquica y con una reducción del stress afectivo. Estos resultados sugerirían que la terapia basada en el "mindfulness" podría ser un abordaje útil en el tratamiento para dejar de fumar que necesitaría ser evaluado en un estudio controlado.

No obstante, y dado lo planteado por algunos modelos conceptuales recientes ${ }^{16,17}$ que tratan de explicar las conductas adictivas sin la presencia de experiencia subjetiva de craving por parte del adicto, surge la siguiente pregunta: ¿cuál es el momento en el que el sujeto deberá poner en práctica las técnicas que le permitan mejorar su "atención consciente" ante una situación de riesgo de recaída? La intervención denominada "prevención de recaídas basada en mindfulness"94 propone la práctica de una serie de técnicas encaminadas al desarrollo de la concienciación y aceptación de los pensamientos, sentimientos y sensaciones para hacer frente a las situaciones de alto riesgo de consumo. La conjunción de estrategias específicas de prevención de recaídas (información acerca del efecto de la violación de la abstinencia, incremento de las expectativas de auto-eficacia, entrenamientos en estrategias de afrontamiento), junto con la práctica regular de "mindfulness" o "atención consciente", pueden dotar al paciente de mayor margen de seguridad para prevenir la recaída ${ }^{95}$. Los pacientes son entrenados en el reconocimiento precoz de las señales de peligro, tanto de estímulos externos como internos, asociados al consumo, así como el incremento de la adopción de un actitud objetiva, de monitorización, de las contingencias que las acompañan. De hecho, se ha observado que las terapias basadas en el "mindfulness" mejorarían las conductas relacionadas con la atención al potenciar subcomponentes específicos de la atención ${ }^{112}$ y específicamente facilitarían la evitación o supresión de pensamientos relacionados con el consumo de sustancias ${ }^{110}$. Por lo tanto, puede considerarse que el "mindfulness" o "atención consciente", en el ámbito de las psicoterapias, es un instrumento con potencial para ejercer un mejor control sobre aquellas conductas problema que queremos manejar. De cualquier modo, son necesarios más estudios, particularmente estudios aleatorizados que permitan valorar la eficacia de dichas técnicas en función de las distintas 
sustancias adictivas y en distintas subpoblaciones de drogodependientes.

Paralelamente, existen autores ${ }^{90}$ que alertan sobre el peligro de la utilización de las técnicas de "mindfulness" o "atención consciente" de forma indiscriminada para cualquier trastorno mental o cualquier perfil de paciente, señalando posibles efectos adversos. La implementación de las estrategias "mindfulness" pueden provocar malestar al requerir un esfuerzo considerable por parte del paciente. Se ha sugerido ${ }^{99}$ que este tipo de técnicas deben ser puestas en tela de juicio ante patologías mentales graves como la esquizofrenia, a la espera de los resultados aportados por estudios que validen con evidencia empírica la oportunidad de su utilización en estos pacientes. Sin embargo, se ha descrito los efectos adversos de algunas técnicas meditativas similares al "mindfulness" en pacientes con historia previa de esquizofrenia ${ }^{113,114}$. Asi mismo, otros autores ${ }^{115,116}$ han observado la aparición de episodios psicóticos en personas sin antecedentes psiquiátricos similares tras períodos de práctica intensiva en las técnicas meditativas. Los trastornos afectivos y los trastornos de ansiedad son otras patologías donde se han encontrado efectos adversos tras la implementación de algunas técnicas meditativas, refiriéndose principalmente incremento de los afectos negativos y de las cogniciones negativas ${ }^{117,}{ }^{118}$. Sin embargo, en el único estudio que hasta la fecha ha evaluado si la gravedad de los síntomas de un trastorno por estrés post-traumático (TEPT) se asociaba con la participación y la evolución del tratamiento en pacientes con un trastorno por uso de sustancias comórbido tratados con MV o mediante tratamiento habitual halló que el grupo que participó en la MV presentaba una reducción significativa en el consumo de sustancias comparado con el grupo control, independientemente de los niveles de gravedad de los síntomas de TEPT ${ }^{119}$. Considerando la elevada prevalencia de otros trastornos mentales comórbidos asociados a los trastornos por uso de sustancias, es necesario ser cautos en todos aquellos pacientes con patología dual a la hora de implementar estrategias basadas en "mindfulness" o "atención consciente", haciéndose necesarios más estudios para evaluar la integración de dichas estrategias en el tratamiento de este perfil de pacientes.

\section{CONCLUSIONES}

Tal como señala el Melbourne Academic Mindfulness Interest Group ${ }^{92}$, existen cuestiones todavía sin resolver referidas al "mindfulness" o "atención consciente": ¿es compatible el "mindfulness" con la farmacoterapia? De hecho en adicciones son muy escasas las evidencias sobre el uso simultáneo de tratamientos farmacológicos en pacientes en tratamiento con terapias basadas en "mindfulness" o "atención consciente". Sin embargo, en pacientes con otros diagnósticos psiquiátricos como es el caso del trastorno por déficit de atención con hiperactividad se ha observado una mayor mejoría con la combinación de su tratamiento psicoestimulante y la terapia de "mindfulness" 120. Otras cuestiones que necesitan ser resueltas se refieren a si es mejor el "mindfulness" en formato de terapia grupal o individual, y si es requisito previo para poder implementar"mindfulness" la práctica personal del terapeuta. Igualmente, sería necesario mayor apoyo empírico para la determinación del peso relativo sobre la eficacia de las técnicas "mindfulness" o "atención consciente" y aquellas técnicas de intervención psicológica o farmacológica que ya han demostrado ser eficaces para la prevención de recaídas.

No obstante, los datos aportados hasta el momento por las investigaciones, apoyarían la hipótesis, sobre la necesidad, aunque no suficiencia, de incrementar en nuestros pacientes la capacidad observacional de sus procesos mentales para disminuir la probabilidad de recaídas. Ello sería posible a través de la implementación de técnicas psicoterapéuticas que posibiliten la puesta en marcha del proceso "focalización atencional selectiva-consciente (no automática)", y que permitan asi mismo la "des-identificación" con sus pensamientos automáticos de craving, ganas compulsivas o deseo hacia la droga.

Este grupo de técnicas bien podrían recibir el nombre de "Terapia Atencional" (TA), definida como el conjunto de estrategias psicoterapéuticas encaminadas a dotar al paciente de la capacidad de distanciamiento, en el episodio de impulsividad o deseo, de aquellos contenidos mentales y emocionales que van a sustentar la toma de decisión para el consumo. La TA no tendría como objetivo terapéutico el cambio de la cognición ni la detención de los pensamientos discriminativos hacia el consumo. La TA estaría encaminada a facilitar la adopción de una postura de observación, de des-identificación del contenido mental propiciatorio de la toma de decisiones hacia la búsqueda de la droga. Entendemos que la capacidad de procesar la información que aparece en la mente del paciente, en el momento del craving, solamente será posible si es capaz de accionar los mecanismos atencionales precisos, es decir, la "focalización atencional selectiva consciente". 


\section{REFERENCIAS}

1. Kozlowski LT, Wilkinson A. Use and misuse of the concept of craving by alcohol, tobacco, and drug researchers. Br J Addict 1987; 82: 31-6.

2. Organización Mundial de la Salud. Décima revisión de la Clasificación Internacional de Enfermedades (CIE-10). Trastornos Mentales y del Comportamiento. Descripciones Clínicas y Pautas para el Diagnóstico. Madrid: Ed Meditor; 1992.

3. Grace A. The tonic/phasic model of dopamine system regulation and its implications for understanding alcohol and psychostimulant craving. Addiction 2000; 95 Supl 2: 119-28.

4. Koob G, Le Moal M. Drug abuse: Hedonic homeostatic dysregulation. Science 1997; 278: 52-8.

5. Robinson TE, Berridge KC. The neural basis of drug craving: an incentive-sensitization theory of addiction. Brain Res Rev 1993; 18: 247-91.

6. Lowman C, Hunt W, Litten R, Drummond C. Research perspectives on alcohol craving: an overview. Addiction 2000; 95 Supl 2: 45-54

7. Littleton J. Can craving be modelled in animals? The relapse prevention perspective. Addiction 2000; 95 Supl 2: 83-90.

8. Larimer ME, Palmer RS, Marlatt A. Relapse prevention. An overview of Marlatt's cognitive-behavioral model. Alcohol Res Health 1999; 23: 151-60.

9. Niaura R. Cognitive social learning and related perspectives on drug craving. Addiction 2000; 95 Supl 2: 155-63.

10. Baker T, Morse E, Sherman J. The motivation to use drugs: A psychobiological analysis of urges. The Nebraska Symposium on Motivation: Alcohol Use and Abuse. Lincoln. NE: University of Nebraska Press; 1987. p. 257-323.

11. May J, Andrade J, Panabokke N, Kavanagh D. Images of desire: cognitive models of craving. Memory 2004; 12: 447-61.

12. Tiffany ST, Conklin CA. A cognitive processing model of alcohol craving and compulsive alcohol use. Addiction 2000; 95 Supl 2: 145-53.

13. Marlatt GA, Gordon JR, editores. Relapse prevention: Maintenance strategies in addictive behavior change. New York: Guildford; 1985.

14. Litman G, Stapleton J, Rawson N, Oppenheimer A, Peleg M, Jackson P. Situations related to alcoholism relapse. Br. J Addict 1983; 78: 381-9.

15. Merikle EP. The subjective experience of craving: an exploratory analysis. Subst Use Misuse 1999; 34: 1101-5.

16. Tiffany ST. A cognitive model of drug urges and druguse behavior: Role of automatic and non-automatic processes. Psychol Rev 1990; 97: 147-68.

17. Tiffany ST. The role of cognitive factors in reactivity to drug cues. En: Drummond DC, Tiffany ST, Glautier SP, Remington B, editores. Addictive Behavior: Cue Exposure Theory and Practice. Chichester, England: John Wiley; 1995. p. 137-65.
18. Ramos J, Tejero A, Trujols J. ¿Craving como elemento central o como epifenómeno en las conductas adictivas? Una revisión de la propuesta de Tiffany. Adicciones 1998; 10: 217-21.

19. Boring EG. A History of Experimental Psychology. New York: Appleton-Century Crofts; 1950.

20. Broadbent DE. Perception and Communication. London: Pergamon Press; 1958.

21. Kahneman D. Attention and Effort. Englewood Cliffts: Prentice-Hall; 1973.

22. Phaf RH, Van der Heijden AH, Hudson PT. SLAM: A connectionist model for attention in visual selection tasks. Cognit Psychol 1990; 22: 273-341.

23. Raz A, Buhle J. Typologies of attentional networks. Nat Rev Neurosci 2006; 7: 367-79.

24. Allport DA. Selection for attention: Some behavioral and neurophysiological considerations on attention and action. En: Heuer H, Sanders AF, editores. Issues on Perception and Action. Hillsdale, NJ: Erlbaum; 1987. p. 395-420.

25. Estevez-Gonzalez A, Garcia-Sanchez C, Junque C. Atención: una función cerebral compleja. Rev Neurol 1997; 25: 1989-97.

26. James W. The Principles of Psychology. New York: Dover; 1890.

27. Pashler HE. The Psychology of Attention. Cambridge, MA: The MIT Press; 1997.

28. Posner MI. Attention: the mechanisms of consciousness. Proc Natl Acad Sci USA 1994; 91: 7398-403.

29. Van der Heijden AH, Bem S. Successive approximations to an adequate model of attention. Conscious Cogn 1997; 6: 413-28.

30. Parasuraman R, Davies DR, editores. Varieties of Attention. Orlando: Academic Press; 1984.

31. Baars BJ. Some essential differences between consciousness and attention, perception, and working memory. Conscious Cogn 1997; 6: 363-71.

32. Norman DA, Shallice T. Attention to action. Willed and automatic control of behavior. En: Davison RJ, Schwartz GE, Shapiro D, editores. Consciouness and Self-regulation (Vol. 4). New York: Plenum Press; 1986. p. 1-18.

33. Reason J. Lapses of attention in everyday life. En: Parasuraman R, Davies DR editores. Varieties of Attention. New York: Academic Press; 1984. p. 515-50.

34. LaBerge D. Attentional control: brief and prolonged. Psychol Res 2002; 66: 220-33.

35. Johnston WA, Dark VJ. Selective attention. Ann Rev Psychol 1986; 37: 43-75.

36. Logan GD. Attention, automaticy and the ability to stop a speeded choice response. En: Long J, Baddeley A, editores. Attention and performance, IX. Hillsdale, NJ: Erlbaum; 1981. p. 205-22.

37. Courtney SM. Attention and cognitive control as emergent properties of information representation in working memory. Cogn Affect Behav Neurosci 2004; 4: 501-16. 
38. Szymura B, Neka E. Visual selective attention and personality: An experimental verification of three models of extraversión. Pers Individ Dif 1998; 25: 713-29.

39. Posner MI, Rothbart MK. Attention, self-regulation and consciousness. Philos Trans R Soc Lond B Biol Sci 1998; 353: 1915-27.

40. Marcel AJ. Conscious and unconscious perception: Experiments on visual masking and word recognition. Cognit Psychol 1983; 15: 197-237.

41. Myers GL, Fisk AD. Training consistent task components: application of automatic and controlled processing theory to industrial task training. Hum Factors 1987; 29 : 255-68.

42. Vecera SP, Rizzo M. Spatial attention: normal processes and their breakdown. Neurol Clin 2003; 21: 575-607.

43. Bargh JA, Ferguson MJ. Beyond behaviorism: on the automaticity of higher mental processes. Psychol Bull 2000; 126: 925-45.

44. Schneider W, Pimm-Smith M, Worden M. Neurobiology of attention and automaticity. Curr Opin Neurobiol 1994; 4: $177-82$

45. Naveh-Benjamin M. Coding of spatial location information: An automatic process? J Exp Psychol Learn Mem Cogn 1987; 13: 595-605.

46. Zbrodroff NJ, Logan CD. On the autonomy of mental processes: A case study of arithmetic. J Exp Psychol Gen 1986; 115: 118-30.

47. Duncan J. Consistent and varied training in the theory of automatic and controlled información processing. Cognition 1986; 23: 279-84.

48. Schneider W, Fisk AD. Automatic category search and its transfer. J Exp Psychol Learn Mem Cogn 1984; 10: $1-15$.

49. Shiffrin RM, Dumais ST. The development of automatism. En: Anderson JR, editor. Cognitive skills and their acquisition. Hillsdale, NJ: Erlbaum; 1981. p. 111-40.

50. Houghton G, Tipper SP. Inhibitory mechanisms of neural and cognitive control: applications to selective attention and sequential action. Brain Cogn 1996; 30: 20-43.

51. Tipper SP, Cranston M. Selective attention and priming: inhibitory and facilitatory effects of ignored primes. Q J Exp Psychol A 1985; 37: 591-611.

52. Wanigaratne S. Psychology of addiction. Psychiatry 2006; 5: 455-60.

53. Witkiewitz K, Marlatt GA. Relapse prevention for alcohol and drug problems: that was Zen, this is Tao. Am Psychol 2004; 59: 224-35.

54. Brownell KD, Marlatt GA, Lichtenstein E, Wilson GT. Understanding and preventing relapse. Am Psychol 1986; 41: 765-82.

55. Marlatt GA, Witkiewitz K. Relapse prevention for alcohol and drug problems. En: Marlatt GA, Donovan DM, editores. Relapse Prevention, Second edition, Maintenance Strategies in the Treatment of Addictive Behaviors. New York: The Guildford Press; 2005. p. 1-44.

56. Marlatt GA. Alcohol, the magic elixir: Stress, expectancy, and the transformation of emotional states. En: Gottheil
E, Druly KA, Pashko S, Weinstein SP, editores. Stress and Addiction. New York: Brunner/Mazel; 1987. p. 302-22.

57. Ryan F. Detected, selected, and sometimes neglected: Cognitive processing of cues in addiction. Exp Clin Psychopharmacol 2002; 10: 67-76.

58. Amato L, Davoli M, Perucci CA, Ferri M, Faggiano F, Mattick RP. An overview of systematic reviews of the effectiveness of opiate maintenance therapies: available evidence to inform clinical practice and research. J Subst Abuse Treat 2005; 28: 321-9.

59. Fiellin DA, Pantalon MV, Chawarski MC, Moore BA, Sullivan LE, O'Connor PG, et al. Counseling plus buprenorphine-naloxone maintenance therapy for opioid dependence. N Engl J Med 2006; 355: 365-74.

60. van den Brink W, Haasen C. Evidenced-based treatment of opioid-dependent patients. Can J Psychiatry 2006; 51: 635-46.

61. Kirchmayer U, Davoli M, Verster A. Naltrexone maintenance treatment for opioid dependence. Cochrane Database Syst Rev 2006; CD001333.

62. Sofuoglu M, Kosten TR. Emerging pharmacological strategies in the fight against cocaine addiction. Expert Opin Emerg Drugs 2006; 11: 91-8.

63. Vocci FJ, Acri J, Elkashef A. Medication development for addictive disorders: The state of the science. Am J Psychiatry 2005; 162: 1432-40.

64. Martinez Raga J, Knecht C, Cepeda S. Modafinil: A useful medication for cocaine addiction? Review of the evidence from neuropharmacological, experimental and clinical studies. CDAR 2008; 1: 213-21.

65. West SL. Pharmacological treatment of alcohol dependence. JAMA 1999; 281: 1318-25.

66. Kleber HD, Weiss RD, Anton RF Jr, George TP, Greenfield SF, Kosten TR, et al. Treatment of patients with substance use disorders, second edition. American Psychiatric Association. Am J Psychiatry 2007; 164 Supl 4: 5-123.

67. Cahill K, Stead LF, Lancaster T. Nicotine receptor partial agonists for smoking cessation. Cochrane Database Syst Rev 2007; CD006103.

68. LancasterT, Stead L, Cahill K. An update on therapeutics for tobacco dependence. Expert Opin Pharmacother 2008; 9: 15-22.

69. Timko C, Finney JW, Moos RH. The 8-year course of alcohol abuse: gender differences in social context and coping. Alcohol Clin Exp Res 2005; 29: 612-21.

70. Cutler RB, Fishbain DA. Are alcoholism treatments effective? The Project MATCH data. BMC Public Health 2005; 5: 75.

71. Dutra L, Stathopoulou G, Basden SL, Leyro TM, Powers MB, Otto MW. A meta-analytic review of psychosocial interventions for substance use disorders. Am J Psychiatry 2008; 165: 179-87.

72. Irvin JE, Bowers CA, Dunn ME, Wang MC. Efficacy of relapse prevention: A meta-analytic review. J Consult Clin Psychol 1999; 67: 563-70. 
73. Roll JM. Contingency management: an evidencebased component of methamphetamine use disorder treatments. Addiction 2007; 102 Supl 1: 114-20.

74. Schottenfeld RS, Chawarski MC, Pakes JR, Pantalon MV, Carroll KM, Kosten TR. Methadone versus buprenorphine with contingency management or performance feedback for cocaine and opioid dependence. Am J Psychiatry 2005; 162: 340-9.

75. McKay JR, Alterman Al, Cacciola JS, Rutherford MJ, O'Brien CP, Koppenhaver J. Group counseling versus individualizad relapse prevention aftercare following intensive outpatient treatment for cocaine dependence: initial results. J Consult Clin Psychol 1997 ; 65: 778-88.

76. Rohsenow DJ, Monti PM, Martin RA, Michalec E, Abrams DB. Brief coping skills treatment for cocaine abuse: 12-month substance use outcomes. J Consult Clin Psychol 2000; 68: 515-20.

77. Higgins ST, Wong CJ, Badger GJ, Haug Ogden DE, Dantona RL. Contingent reinforcement increases abstinence during outpatient treatment and 1 year of follow-up. J Consult Clin Psychol 2000; 68: 64-72.

78. Silverman K, Chutuape MA, Bigelow GE, Stitzer ML. Voucher-based reinforcement of cocaine abstinence in treatment-resistant methadone patients: effects of reinforcement magnitude. Psychopharmacology 1999; 146: 128-38.

79. Guichenez P, Clauzel I, Cungi C, Quantin X, Godard P, Clauzel AM. The contribution of cognitive behavioural therapies to smoking cessation. Rev Mal Respir. 2007; 24: 171-82.

80. Holder HD, Cisler RA, Longabaugh R, Stout RL, Treno AJ, Zweben A. Alcoholism treatment and medical care costs from Project MATCH. Addiction 2000; 95: 999 1013.

81. Irvin JE, Bowers CA, Dunn ME, Wang MC. Efficacy of relapse prevention: A meta-analytic review. J Consult Clin Psychol 1999; 67: 563-70.

82. Carroll KM, Rounsaville BJ, Gordon LT, Nich C, Jatlow PM, Bisighini RM, et al. Psychotherapy and pharmacotherapy for cocaine abusers. Arch Gen Psychiatry 1994; 51: 177-87.

83. Maude-Griffin PM, Hohenstein JM, Humfleet GL, Reilly PM, Tusel DJ, Hall SM. Superior efficacy of cognitivebehavioral therapy for urban crack cocaine abusers: main and matching effects. J Consult Clin Psychol 1998; 66: 832-37.

84. Hawkins JD, Catalano RF Jr, Gillmore MR, Wells EA. Skills training for drug abusers: generalization, maintenance, and effects on drug use. J Consult Clin Psychol 1989; 57: 559-63.

85. Woody GE, McLellan AT, Luborsky L, O'Brien CP. Twelve-month follow-up of psychotherapy for opiate dependence. Am J Psychiatry 1987; 144: 590-6.

86. Baer RA. Mindfulness training as a clinical intervention: a conceptual and empirical review. Clin Psychol 2003; 10: $125-43$
87. Krisanaprakornkit T, Krisanaprakornkit W, Piyavhatkul N, Laopaiboon M. Meditation therapy for anxiety disorders. Cochrane Database Syst Rev 2006; CD004998.

88. Allen NB, Chambers R, KnightW, Melbourne Academic Mindfulness Interest Group. Mindfulness-based psychotherapies: a review of conceptual foundations, empirical evidence and practical considerations. Aust N Z J Psychiatry 2006; 40: 285-94.

89. Bishop SR, Lau M, Shapiro S, Carlson L, Anderson ND, Carmody J, et al. Mindfulness: A proposed operational definition. Clin Psychol 2004; 11: 230-41.

90. Kabat-Zinn J. Mindfulness-based interventions in context: past, present, and future. Clin Psychol 2003; 10: $144-56$

91. Leigh J, Bowen S, Marlatt GA. Spirituality, mindfulness and substance abuse. Addict Behav 2005; 30: 1335-41.

92. Kabat-Zinn J. Wherever you go, there you are: mindfulness meditation in everyday life. New York: Hyperion; 1994.

93. Teasdale JD, Segal ZV, Williams JM, Ridgeway VA, Soulsby JM, Lau MA. Prevention of relapse/recurrence in major depression by mindfulness-based cognitive therapy. J Consult Clin Psychol 2000; 68: 615-23.

94. Witkiewitz K, Marlatt GA, Walter D. Mindfulness-based relapse prevention for alcohol and substance use disorders. J Cogn Psychother 2005; 19: 211-28.

95. Curtis F, Zack M, McMain S. An information-processing analysis of mindfulness: implications for relapse prevention in the treatment of substance abuse. Clin Psychol Sci Prac 2002; 9: 275-99.

96. Kabat-Zinn J, Massion AO, Kristeller J, Peterson L, Fletcher KE, Pbert L. Effectiveness of a meditationbased stress reduction program in the treatment of anxiety disorders. Am J Psychiatry 1992; 149: 936-43.

97. Grossman P, Niemann L, Schmidt S, Walach H. Mindfulness-based stress reduction and health benefits. A meta-analysis. J Psychosom Res 2004; 57: 35-43.

98. Linehan M. Cognitive-behavioral treatment of borderline personality disorder. New York: Guildford Press; 1993.

99. Segal ZV, Williams JMG, Teadsle J. Mindfulness-based cognitive therapy for depression: a new approach to preventing relapse. New York: Guildford, 2002.

100. Hayes SC, Strosahl KD, Wilson KG. Acceptance and commitment therapy. New York: Guildford Press; 1999.

101. Buccheld N, Grossman P, Walach H. Measuring mindfulness in insight meditation (Vipassana) and meditation-based psychotherapy: The development of the Freiburg Mindfulness Inventory (FMI). J Medit Medit Res 2001; 1: 11-34.

102. Brown KW, Ryan RM. The benefits of being present: Mindfulness and its role in psychological well-being. J Pers Soc Psychol 2003; 84: 822-48.

103. Baer RA, Smith GT, Allen KB. Assessment of mindfulness by self-report: The Kentucky Inventory of Mindfulness Skills. Assessment 2004; 11: 191-206.

104. Feldman, GC, Hayes AM, Kumar SM, Kamholz BW, Greeson JM, Laurenceau J. Assessing mindfulness 
in the context of emotion regulation: The Revised Cognitive and Affective Mindfulness Scale (CAMS-R). Comunicación presentada a la $39^{\text {th }}$ Annual Convention of the Association for Behavioral and Cognitive Therapies. 2005. Washington, DC, Noviembre de 2005.

105. Cardaciotto L, Herbert JD. The development of a bi-dimensional measure of mindfulness: The Philadelphia Mindfulness Scale (PHLMS). Comunicación presentada a la 39th Annual Convention of the Association of Behavioral and Cognitive Therapies. 2005. Washington, DC, Noviembre de 2005.

106. Lau MA, Bishop SR, Segal ZV, Buis T, Anderson ND, Carlson L, et al. The Toronto Mindfulness Scale: development and validation. J Clin Psychol 2006; 62: 1445-67.

107. Alterman Al, Koppenhaver JM, Mulholland E, Ladden $L J$, Baime MJ. Pilot trial of effectiveness of mindfulness meditation for substance abuse patients. J Subst Use 2004; 9: 259-68.

108. Bowen S, Witkiewitz K, Dillworth TM, Charla N, Simpson TL, Ostafin BD, et al. Mindfulness meditation and substance use in an incarcerated population. Psychol Addict Behav 2006; 20: 343-7.

109. Davis JM, Fleming MF, Bonus KA, Baker TB. A pilot study on mindfulness based stress reduction for smokers. BMC Complement Altern Med 2007; 7: 2.

110. Bowen S, Witkiewitz K, Dillworth TM, Marlatt GA. The role of thought suppression in the relationship between mindfulness meditation and alcohol use. Addict Behav 2007; 32: 2324-8.
111. Shafil M, Lavely R, Jaffe R. Meditation and the prevention of alcohol abuse. Am J Psychiatry 1975; 132: 942-5.

112. Jha AP, Krompinger J, Baime MJ. Mindfulness training modifies subsystems of attention. Cogn Affect Behav Neurosci 2007; 7: 109-19.

113. Garcia R, Monterrey AL, Gonzalez de Rivera JR. Meditación y psicosis. Psiquis 1992; 13: 39-43.

114. Walsh R, Roche L. Precipitation of acute psychotic episodes by intensive meditation in individuals with a history of schizophrenia. Am J Psychiatry 1979; 136: 1085-6.

115. Sethi S, Bhargava SC. Relationship of meditation and psychosis: case studies. Aust N Z J Psychiatry 2003; 37: 382.

116. Chan-OB T, Boonyanaruthee V. Meditation in association with psychosis. J Med Assoc Thai 1999; 82: 925-30.

117. Otis SL. The facts on transcendental meditation: III. If well-integrated but anxious, try MT. Psychol Today 1974; 7: 45-6.

118. Lazarus AA. Psychiatric problems precipitated by Transcendental Meditation. Psychol Rep 1976; 39: 601-2.

119. Simpson TL, Kaysen D, Bowen S, MacPherson LM, Chawla N, Blume A, et al. PTSD symptoms, substance use, and vipassana meditation among incarcerated individuals. J Trauma Stress 2007; 20: 239-49.

120. Zylowska L, Ackerman DL, Yang MH, Futrell JL, Horton NL, Hale TS, et al. Mindfulness meditation training in adults and adolescents with ADHD: A feasibility study. J Atten Disord 2008; 11: 737-46. 